\title{
Diffuse idiopathic skeletal hyperostosis is associated with lumbar spinal stenosis requiring surgery
}

Kentaro Yamada, Shigenobu Satoh, Hiroshi Hashizume, Noriko Yoshimura, Ryohei Kagotani, Yuyu Ishimoto, Yuichiro Abe, Hiromitsu Toyoda, Hidetomi Terai, Takeshi Masuda, Shigeyuki Muraki, Hiroaki Nakamura, Munehito Yoshida

\begin{tabular}{|c|l|}
\hline Citation & Journal of Bone and Mineral Metabolism, 37(1); 118-124 \\
\hline Issue Date & $2019-01$ \\
\hline Type & Journal Article \\
\hline Textversion & author \\
\hline Relation & $\begin{array}{l}\text { This is a post-peer-review, pre-copyedit version of an article published in General } \\
\text { Thoracic and Cardiovascular Surgery. The final authenticated version is available } \\
\text { online at: } \text { https://doi.org/10.1007/s00774-017-0901-0 }\end{array}$ \\
\hline DOI & $10.1007 /$ s00774-017-0901-0 \\
\hline
\end{tabular}

\author{
Self-Archiving by Author(s) \\ Placed on: Osaka City University
}

YAMADA K, et al. (2019). Diffuse idiopathic skeletal hyperostosis is associated with lumbar spinal stenosis requiring surgery. Journal of Bone and Mineral Metabolism. 37, 118-124. 


\section{Abstract}

Factors related to the onset and progression of lumbar spinal stenosis (LSS) have not yet been identified. Diffuse idiopathic skeletal hyperostosis (DISH) increases mechanical loading on the non-fused lumbar levels and may therefore lead to LSS. This cross-sectional study was aimed to identify associations between LSS and DISH. This study included 2363 consecutive patients undergoing surgery for LSS and 787 general inhabitants without symptoms of LSS as participants of the population-based cohort study, Research on Osteoarthritis/Osteoporosis Against Disability (ROAD). Standing whole-spine radiographs were used to diagnose DISH based on the criteria proposed by Resnick. The prevalence of DISH showed step wise increase significantly among asymptomatic inhabitants without radiographic LSS, asymptomatic inhabitants with radiographic LSS, and LSS patients required surgery $(14.4 \%, 21.1 \%$, and $31.7 \%, p<0.001)$. The distribution of DISH was similar between the groups, but the lower thoracic and upper-middle lumbar spine regions were more frequently involved in patients with LSS required surgery. Multivariate analysis indicated DISH was an independent associated factor for LSS required surgery (adjusted odds ratio 1.65, 95\% confidence interval 1.32-2.07), after adjustment for age, sex, body mass index, and diabetes mellitus. Among patients with LSS required surgery, higher occurrence of stenosis at upper lumbar levels and multi- level stenosis were observed in patients with DISH required surgery than without DISH. In 
conclusion, DISH is independently associated with LSS required surgery. The decrease in the lower mobile segments by DISH may increase the onset or severity of LSS.

Key Words: Diffuse idiopathic skeletal hyperostosis, Lumbar spinal stenosis, General inhabitants, Prevalence, Standing whole-spine radiographs 


\section{Diffuse Idiopathic Skeletal Hyperostosis is Associated With Lumbar Spinal}

\section{Stenosis required surgery}

Kentaro Yamada ${ }^{1,2 *}$, Shigenobu Satoh ${ }^{1}$, Hiroshi Hashizume ${ }^{3}$, Noriko Yoshimura ${ }^{4}$,

Ryohei Kagotani ${ }^{3}$, Yuyu Ishimoto ${ }^{3}$, Yuichiro Abe ${ }^{1}$, Hiromitsu Toyoda ${ }^{2}$, Hidetomi

Terai $^{2}$, Takeshi Masuda ${ }^{1}$, Shigeyuki Muraki ${ }^{4}$, Hiroaki Nakamura ${ }^{2}$, Munehito Yoshida 3

1 Department of Orthopaedic Surgery, Wajokai Eniwa Hospital, Hokkaido, Japan

2 Department of Orthopaedic Surgery, Osaka City University, Osaka, Osaka, Japan

3 Department of Orthopaedic Surgery, Wakayama Medical University, Wakayama,

Japan

4 Department of Joint Disease Research, 22nd Century Medical and Research Center,

Faculty of Medicine, The University of Tokyo, Tokyo, Japan

*Corresponding author: Kentaro Yamada, MD, PhD 
Department of Orthopaedic Surgery, Osaka City University Graduate School of Medicine

1-4-3 Asahi-machi, Abeno-ku, Osaka 545-8585, Japan

Tel: +81-6-6645-3851, Fax: +81-6-6646-6260

E-mail address: yamachen@msic.med.osaka-cu.ac.jp

Key Words: Diffuse idiopathic skeletal hyperostosis, Lumbar spinal stenosis, General inhabitants, Prevalence, Standing whole-spine radiographs 


\section{Introduction}

Lumbar spinal stenosis (LSS) is a chronic disease defined as the radiographic narrowing of the lumbar canal or neural foramen with encroachment on neural structures by the surrounding bone and soft tissue. LSS is also characterized by degenerated facet joints, disc protrusion and hypertrophy of the ligamentum flavum.[1] The clinical symptoms of LSS in the elderly include impaired walking and other disabilities.[1] However, severe radiographic LSS is often observed in asymptomatic patients.[2] A population-based cohort study previously performed by our group found that the prevalence of symptomatic LSS was only $17.5 \%$ of participants with severe stenosis which observed 30.4\% among the general population.[3] Although advanced age, diabetes mellitus (DM), urological disorders, hypertension, osteoarthritis/fractures, and depressive symptoms are reportedly likely to be observed in patients with symptomatic LSS,[4,5] the triggers for LSS symptoms in patients with radiographic LSS remain unclear.

Diffuse idiopathic skeletal hyperostosis (DISH) is a noninflammatory skeletal disease of unknown etiology characterized by calcification and ossification of soft tissues, predominantly ligaments and entheses. In DISH patients, the spinal longitudinal ligaments and entheses slowly become ossified and show decreased mobility in the 
affected region until complete ankylosis results.[6] DISH begins most frequently in the lower thoracic spinal segments, and later extends into the upper thoracic segments and lumbar spine.[7] The lumbosacral segment is exposed to higher mechanical stress due to fusion of thoracolumbar vertebral segments in patients with than without DISH.

Therefore, DISH could be a trigger for the onset or increased severity of LSS, and some studies have indicated that mechanical stress causes accelerated ligamentum flavum hypertrophy and leads to LSS.[8] However, except in a few case reports of DISH patients who exhibited LSS, $[9,10]$ there have been no detailed analyses of the association between LSS and DISH. Here, we hypothesized that a positive correlation exists between symptomatic LSS required surgery and DISH.

The aim of this study was to investigate the prevalence of DISH, using a standardized definition of DISH in a cross-sectional study, in patients with LSS required $\underline{\text { surgery }}$ and subjects without symptom of LSS, and to clarify the association between $\underline{\text { LSS required surgery and DISH using multivariate analysis that accounted for potential }}$ confounding factors.

\section{Materials and Methods}




\section{Subjects}

This study included patients undergoing surgery for symptomatic LSS and general inhabitants without LSS. This study was approved by the institutional review board (IRB) of the Wajokai Eniwa Hospital and Wakayama Medical University. Patients undergoing surgery were consecutive patients identified from the database of surgery for LSS during specific periods in which preoperative whole-spine radiographs were routinely taken at one institution (Eniwa, Hokkaido, Japan). All patients exhibited symptoms of LSS and required surgery. The surgical indications for LSS were as follows: symptoms of neurogenic intermittent claudication, intolerable leg pain or numbness refractory to conservative treatment, severe muscle weakness, or bladder or bowel dysfunction. The present study included LSS accompanied by spondylolisthesis, lumbar scoliosis, and disc herniation as well as simply degenerated facet joints, disc protrusion, or hypertrophy of the ligamentum flavum. Patients with prior spinal surgery, acute vertebral fracture, spinal malignant neoplasm, spinal infection, general inflammatory diseases such as rheumatoid arthritis or ankylosing spondylitis, and younger patients ( $<50$ years) were excluded. Eighteen patients were excluded because of a lack of radiographs. Fifty-two patients were excluded because of 
difficulty interpreting radiographs. Thus, 2363 patients were included in the present study as patients with LSS required surgery.

The general inhabitant participants were part of the Wakayama Spine Study, which assessed a sub-cohort drawn from Research on Osteoarthritis/Osteoporosis Against Disability (ROAD), a large-scale, prospective study of bone and joint diseases among population-based cohorts established in several communities throughout Japan.[11] We registered 1011 participants from the Wakayama Spine Study to participate in a second survey of the ROAD study, which investigated clinical and radiolographic examination for LSS.[3] Participants in the Wakayama Spine Study were inhabitants of the mountainous region of Hidakagawa and the coastal region of Taiji in Wakayama. The medical history and physical examination for LSS were conducted by an experienced orthopedic surgeon (Y.I.). Assessment of clinical symptoms was based on the definition of LSS in the North American Spine Society guidelines,[12] and diagnosis of LSS required one or more of the following symptoms: pain, numbness, and neurological deficits in the lower extremities and buttocks, and bladder or bowel dysfunction. The diagnosis of LSS required that symptoms be induced or exacerbated by walking or prolonged standing and relieved by lumbar flexion, sitting, and recumbency. Radiographic LSS were applied the classification included in a general 
guideline [13] in which severe stenosis as narrowing of more than two-thirds of the central area on the axial images of magnetic resonance imaging. Participants with prior spinal surgery, younger participants ( $<40$ years), and those for whom whole-spine radiographs could not be obtained were the first excluded from the present study. The remaining 868 participants were recruited, and 81 participants were excluded because of symptoms of LSS. Thus, 787 participants were included as inhabitants without LSS.

\section{Evaluation}

Two authors (K.Y. and R.K.), who were blinded to patients' clinical information, diagnosed DISH on the basis of criteria defined by Resnick and Niwayama[7]: at least four contiguous vertebral segments, preservation of intervertebral disc spaces, and the absence of apophyseal joint ankylosis or sacroiliac inflammatory changes using whole-spine radiographs. The fused vertebrae by DISH were investigated. Fifty random participants were measured twice by one author (K.Y.) at a 1-month interval, and by two authors (K.Y. and R.K.) independently. Intra-class and inter-class correlation coefficients were 0.831 and 0.935 , respectively. Preoperative data, surgical level, and surgical procedures were taken from patient records in the patients with LSS 
required surgery. Height and body weight, which was measured on a digital scale with the participant wearing light clothing and no shoes, were used to calculate body mass

index $\left(\mathrm{BMI}, \mathrm{kg} / \mathrm{m}^{2}\right)$. DM was defined as positive in patients who were under treatment for DM or who had a hemoglobin A1c of $>6.5 \%$.

\section{Statistical analysis}

Differences between the two groups for categorical variables and continuous variables were examined using the chi-square test and the Mann-Whitney U test, respectively. A p-value of $<0.05$ was considered statistically significant. To account for factors associated with symptomatic LSS, a multiple logistic regression model was used to obtain adjusted odds ratios (aORs) with $95 \%$ confidence intervals (CIs). Factors included in the multivariate model were age, sex, BMI, DM, and DISH. All statistical analyses were performed using IBM SPSS Statistics for Windows, Version 23.0 (IBM Corp., Armonk, NY, USA).

\section{Results}


DISH involvement between patients with LSS required surgery and subjects without

\section{$\underline{\text { LSS symptom }}$}

DISH was observed in 750 LSS patients required surgery and 128 inhabitants

without LSS symptom. The prevalence of DISH was significantly higher in patients

with LSS required surgery $(31.7 \%)$ than in inhabitants without LSS symptom (16.3\%)

( $\mathrm{p}<0.001)$. In addition to DISH prevalence, there were significant differences in

patients' characteristics, including sex distribution, BMI, and DM between the groups

(Table 1). Among inhabitants without LSS symptom, radiographic severe LSS at least one level was observed in $223(28.4 \%)$ inhabitants. The prevalence of DISH differed

significantly between inhabitants with radiographic LSS and those without severe

radiographic LSS ( 47 cases $21.1 \%$ and 81 cases $14.4 \%$, respectively, $\mathrm{p}=0.023$, Figure

1).

Fig2. shows the distribution of DISH-affected vertebrae in patients with $\underline{\mathrm{LSS}}$

required surgery and inhabitants without LSS. The distribution of DISH-related

vertebral fusion was similar, exhibiting a platykurtic distribution between the two

groups, However, fused vertebrae by DISH observed significantly caudal in

symptomatic LSS patients than general inhabitants (median T9 and T8, respectively, $\mathrm{p}$ 
$\leq 0.001$ ). The number of fused segments did not differ significantly (symptomatic LSS

6.49 [2.50], inhabitants without LSS 6.94 [3.17], mean [standard deviation], $\mathrm{p}=0.367$ ).

Comparison between patients with LSS required surgery and subjects without LSS

symptom

To investigate LSS required surgery and DISH, multivariate analysis was

performed with adjustment for potential confounders (age, sex, BMI, and DM).

Multiple logistic regression analysis showed that male sex (aOR, 1.66; 95\% CI, 1.39-

1.98), a higher BMI (aOR, 1.15; 95\% CI, 1.12-1.18), DM (aOR, 3.09; 95\% CI, $2.18-$

4.37), and DISH (aOR, 1.65; 95\% CI, 1.32-2.07) were independent risk factors for LSS

required surgery (Table 2).

Association DISH and decompression levels in patients with LSS required surgery.

The association between lower end vertebra of DISH and stenotic levels by

$\underline{\text { LSS were investigated in patients with LSS required surgery. Decompression levels at }}$

$\underline{\text { surgery were investigated as stenotic levels because all surgeries were performed }}$

decompression with/without additional fusion for stenosis. 
Fig 3 (A) shows differences between patients with and without DISH regarding

decompression levels. L4-5 level were most affected levels in both group, however, upper lumbar lesion (L1-2 and L2-3) were more performed decompression in patient with DISH than those without DISH $(\mathrm{P}<0.001)$. Moreover, the multi-level stenosis which required $\geq 3$ levels decompression were more frequent in patients with DISH (169 pts, 39.2\%) than those without DISH (581 pts, 30.0\%, p <0.001). Fig 3 (B) shows the stenosis on just distal of the fused vertebrae were observed in 30 of $750(4 \%)$.

\section{Discussion}

The present study revealed that DISH is independently associated with $\underline{\text { LSS }}$ required surgery. DISH was first described by Forestier and Rotes-Querol in 1950, at which time the disease was called senile ankylosing hyperostosis.[14] DISH is currently diagnosed based on the classification criteria established by Resnick and Niwayama.[7] The complications of DISH include unstable spinal fractures, dysphagia, postsurgical heterotopic ossification, difficult intubation, difficult gastroscopy, aspiration pneumonia, and myelopathy.[15,16] Although we could not evaluate cause and effect in this cross-sectional study, LSS cannot cause DISH as a noninflammatory skeletal disease. 
Therefore, our results show that LSS is a complication of DISH in terms of onset or increased severity. To the best of our knowledge, this is the first study to investigate associations between DISH and LSS in a large population while adjusting for multiple confounding factors.

Although the mechanism of the positive association between DISH and LSS is still unclear, a likely reason is the increased mechanical load secondary to DISH-induced spinal segment fusion. Fractures of an ankylosed spine in patients with DISH tend to be unstable even after low-energy impact and can result in neurological deficits compared with a normal spine.[16] These poor clinical outcomes after trauma are believed to be caused by the development of long lever arms in the spinal column, upon which forces can act during trauma, resulting in instability of fracture site/non-fused segments caused by ossification of supportive and elastic soft tissues. Meanwhile, DISH-induced spinal segment fusion may also chronically magnify the biomechanical load at the non-fused segment, as in patients with acute spinal trauma. Therefore, the increased stress associated with hypermobility of the non-fused spinal segment in patients with DISH could cause disc degeneration or hypertrophy of the ligamentum flavum and lead to LSS. The step wise increase of prevalence of DISH among the group (asymptomatic inhabitants without radiographic LSS 14.4\%, 
asymptomatic inhabitants with radiographic severe LSS $21.1 \%$, and patients required

surgery for LSS symptom $31.7 \%$ ) supported our hypothesis in one aspect.

In the present study, there are a few patients required decompression at just

distal level of fused segment by DISH. The most affected stenotic level were lower

lumbar segment which were generally exposed highest stress during postural

change[17,18], even patients with DISH. However, higher occurrence of stenosis at

upper lumbar levels, and multi- level stenosis were observed in patients with DISH

required surgery than without DISH in patients with LSS required surgery. This

tendency may indicate that the presence of fewer mobile intervertebral segments in the

middle-lower lumbosacral spine increases the potential for LSS requiring surgery

because of greater mechanical loading.

Kyphosis may play a role in LSS manifestation and presentation. Hirano et

al.[19] found that patients with kyphotic posture were more likely to have symptomatic

LSS. Kyphosis of the thoracic or lumbar spine has frequently been observed in patients

with DISH.[10,20] When considering these reports and the results of the present study, the increased mechanical stress in patients with DISH might trigger LSS symptoms in patients with radiographic LSS, coupled with kyphotic spinal alignment. 
Recent studies have also suggested an association of several genetic factors (COL6A, FGF2, or ENT1), with DISH.[21,22] Although an association between these genes and the development of LSS has not been reported, future studies may identify the gene common to both abnormal ossification and LSS, but the clinical and basic mechanisms will also need to be clarified.

The reported prevalence of DISH varies widely, from $2.9 \%$ to $39 \%$.[10,23-26] This wide range may be due to differences among investigated populations and screening methods.

Prior studies have associated DISH with demographic and metabolic factors such as race,[23,24] male sex,[10,23,24,26] advanced age,[10,23,24,26] obesity,[14,27] and DM.[27] We previously evaluated patients for LSS using whole-spine radiographs and found the greatest prevalence of DISH (52/132 participants; 39.4\%) in LSS patients who were $\geq 40$ years old.[10] The prevalence in the previous study was greater than $10.8 \%$, which was shown in our report of the first survey of the ROAD study that also used whole-spine radiographs in the Japanese general population (without evaluation for LSS).[26] However, in the previous study, the number of participants was too small for the prevalence of DISH to be determined. In the present study, we investigated a sufficient number of patients with LSS at one institution and confirmed a high 
prevalence $(31.7 \%)$ of DISH in patients with LSS. Also, we directly compared that result with the prevalence in the general population without LSS, detected in the Wakayama Spine Study, to confirm that DISH had a positive influence on symptomatic LSS with adjustment for potential confounding factors.

The majority of reports used lateral chest radiographs to diagnose DISH.[23,24] Although Mata et al.[28] demonstrated high reliability of chest radiographs for the diagnosis of DISH, chest radiographs cannot evaluate the cervical or lumbar region. A whole-spine radiograph can evaluate all spinal segments except lower cervical to upper thoracic, which are obscured by the scapulae or humeral heads. The reliability for the diagnosis of DISH was almost perfect in this study (intra- and inter-class correlation coefficients of 0.831 and 0.935 , respectively). Therefore, the prevalence of DISH demonstrated in this study can be considered accurate with respect to past reports.

There were several limitations to this study. First, the severity of LSS was not considered in the analysis because the evaluations of severity of LSS differed by institution. Although the Wakayama Spine Study included the modified Zurich Claudication Questionnaire to evaluate the severity of LSS, the sample size of symptomatic LSS patients in the general population $(n=81)$ was too small to allow a 
relationship between DISH and LSS symptom severity to be determined. This could be addressed in a larger study. Second, Several factors which related to LSS symptom severity, for example; spinal disorder such as spondylolisthesis [29]/degenerative scoliosis[1] or psychological factors[5], were not included in this study. Although no study has investigated the association between DISH and depression, patients' psychological status can affect the results. Future studies should include these confounders by matched case-control studies. Third, there were geographical differences in this study. The patients with LSS required surgery were recruited from all over Hokkaido, whereas the participants from the general population were recruited from Wakayama. Racial and ethnic differences have been suggested as important predisposing factors for DISH.[23,24] The race and culture between Hokkaido and Wakayama are almost identical, but the food slightly differs because the distance between these two regions is approximately $1200 \mathrm{~km}$. To confirm the reproducibility in a different regional setting, the same analyses were conducted between the general population without LSS in Wakayama and patients with LSS required surgery in Osaka, which is located near Wakayama in the Kinki region of central Japan. This analysis provided the same results regarding the impact of DISH on LSS; DISH was an independent associated factor for LSS required surgery (Supplemental data 1). Hence, 
the effect on the results of the regional difference seen in the present study is likely negligible.

In conclusion, this is the first report to demonstrate a significant association between the presence of DISH and LSS required surgery. DISH was an independent associated risk factor for LSS required surgery after adjustment for age, sex, BMI, and DM. The decrease in the number of mobile segments caused by DISH-induced vertebral fusion may increase the onset or severity of LSS. DISH is diagnosed not only by orthopedic surgeons and neurologists, but also by rheumatologists and general practitioners using plain radiographs. Thus, an awareness of the high prevalence of LSS in patients with DISH can lead to early diagnosis and treatment.

\section{Acknowledgements}

The authors wish to thank Dr. Shinji Takahashi, MD, PhD, for statistical assistance. The authors also thank Ms. Tomoko Takijiri and other members of the Public Office in Hidakagawa Town, and Ms. Tamako Tsutsumi, Ms. Kanami Maeda, and other members 
of the Public Office in Taiji Town, for their assistance in locating and scheduling

participants for examinations for the Wakayama Spine Study.

\section{Conflicts of Interest and Source of Funding}

This study was supported by Grants-in-Aid for Scientific Research (B20390182,

B23390357, B26860419, C20591737, C20591774, and C26462249), for Young

Scientists (A18689031), and for Exploratory Research (19659305) from the Japanese

Ministry of Education, Culture, Sports, Science and Technology; H17-Men-eki-009,

H18-Choujyu-037, and H20-Choujyu-009 from the Ministry of Health, Labour and

Welfare; Research Aid from the Japanese Orthopaedic Association; grants from the

Japanese Orthopaedics and Traumatology Foundation, Inc. (Nos. 166 and 256); and a

Grant-in-Aid for Scientific Research (C22591639) from the Japanese Society for the

Promotion of Science. The sponsors had no role in the study design, data collection, data analysis, data interpretation, or writing of the report.

\section{References}


1. Katz JN, Harris MB (2008) Clinical practice. Lumbar spinal stenosis. N Engl J Med 358:818-825

2. Boden SD, Davis DO, Dina TS, Patronas NJ, Wiesel SW (1990) Abnormal magnetic-resonance scans of the lumbar spine in asymptomatic subjects. A prospective investigation. J Bone Joint Surg Am 72:403-408

3. Ishimoto Y, Yoshimura N, Muraki S, Yamada H, Nagata K, Hashizume H, Takiguchi N, Minamide A, Oka H, Kawaguchi H, Nakamura K, Akune T, Yoshida M (2013) Associations between radiographic lumbar spinal stenosis and clinical symptoms in the general population: the Wakayama Spine Study. Osteoarthritis Cartilage 21:783-788

4. Uesugi K, Sekiguchi M, Kikuchi S, Konno S (2013) Relationship between lumbar spinal stenosis and lifestyle-related disorders: a cross-sectional multicenter observational study. Spine (Phila Pa 1976) 38:E540-545

5. Yabuki S, Fukumori N, Takegami M, Onishi Y, Otani K, Sekiguchi M, Wakita T, Kikuchi S, Fukuhara S, Konno S (2013) Prevalence of lumbar spinal stenosis, using the diagnostic support tool, and correlated factors in Japan: a population-based study. J Orthop Sci 18:893-900 
6. Yaniv G, Bader S, Lidar M, Herman A, Shazar N, Aharoni D, Eshed I (2014)

The natural course of bridging osteophyte formation in diffuse idiopathic skeletal hyperostosis: retrospective analysis of consecutive CT examinations over 10 years. Rheumatology (Oxford) 53:1951-1957

7. Resnick D, Niwayama G (1976) Radiographic and pathologic features of spinal involvement in diffuse idiopathic skeletal hyperostosis (DISH). Radiology 119:559-568

8. Sairyo K, Biyani A, Goel V, Leaman D, Booth R, Jr., Thomas J, Gehling D, Vishnubhotla L, Long R, Ebraheim N (2005) Pathomechanism of ligamentum flavum hypertrophy: a multidisciplinary investigation based on clinical, biomechanical, histologic, and biologic assessments. Spine (Phila Pa 1976) $30: 2649-2656$

9. Karpman RR, Weinstein PR, Gall EP, Johnson PC (1982) Lumbar spinal stenosis in a patient with diffuse idiopathic skeletal hypertrophy syndrome. Spine (Phila Pa 1976) 7:598-603

10. Yamada K, Toyoda H, Terai H, Takahashi S, Nakamura H (2014) Spinopelvic alignment of diffuse idiopathic skeletal hyperostosis in lumbar spinal stenosis. Eur Spine J 23:1302-1308 
11. Yoshimura N, Muraki S, Oka H, Kawaguchi H, Nakamura K, Akune T (2010) Cohort profile: research on Osteoarthritis/Osteoporosis Against Disability study. Int J Epidemiol 39:988-995

12. North American Spine Society Clinical Guidelines. Diagnosis and Treatment of Degenerative Lumbar Spinal Stenosis https://www.spine.org/Portals/0/Documents/ResearchClinicalCare/Guidelines/L umbarStenosis.pdf. Accessed June 092016

13. Suri P, Rainville J, Kalichman L, Katz JN (2010) Does this older adult with lower extremity pain have the clinical syndrome of lumbar spinal stenosis? JAMA 304:2628-36.

14. Forestier J, Rotes-Querol J (1950) Senile ankylosing hyperostosis of the spine. Ann Rheum Dis 9:321-330

15. Mader R (2002) Clinical manifestations of diffuse idiopathic skeletal hyperostosis of the cervical spine. Semin Arthritis Rheum 32:130-135

16. Westerveld LA, Verlaan JJ, Oner FC (2009) Spinal fractures in patients with ankylosing spinal disorders: a systematic review of the literature on treatment, neurological status and complications. Eur Spine J 18:145-156 
17. Nachemson AL (1981) Disc pressure measurements. Spine (Phila Pa 1976)

6:93-97

18. Putzer M, Auer S, Malpica W, Suess F, Dendorfer S (2016) A numerical study

to determine the effect of ligament stiffness on kinematics of the lumbar spine

during flexion. BMC Musculoskelet Disord 17:95

doi:10.1186/s12891-016-0942-X

19. Hirano K, Imagama S, Hasegawa Y, Muramoto A, Ishiguro N (2013) Impact of spinal imbalance and BMI on lumbar spinal canal stenosis determined by a diagnostic support tool: cohort study in communityliving people. Arch Orthop Trauma Surg 133:1477-1482

20. Nardo L, Lane NE, Parimi N, Cawthon PM, Fan B, Shepherd J, Cauley J, Zucker-Levin A, Murphy RA, Katzman WB (2014) Diffuse idiopathic skeletal hyperostosis association with thoracic spine kyphosis: a cross-sectional study for the Health Aging and Body Composition Study. Spine (Phila Pa 1976) 39:E1418-1424

21. Tsukahara S, Miyazawa N, Akagawa H, Forejtova S, Pavelka K, Tanaka T, Toh S, Tajima A, Akiyama I, Inoue I (2005) COL6A1, the candidate gene for ossification of the posterior longitudinal ligament, is associated with diffuse 
idiopathic skeletal hyperostosis in Japanese. Spine (Phila Pa 1976)

$30: 2321-2324$

22. Warraich S, Bone DB, Quinonez D, Ii H, Choi DS, Holdsworth DW, Drangova M, Dixon SJ, Seguin CA, Hammond JR (2013) Loss of equilibrative nucleoside transporter 1 in mice leads to progressive ectopic mineralization of spinal tissues resembling diffuse idiopathic skeletal hyperostosis in humans. J Bone Miner Res 28:1135-1149

23. Weinfeld RM, Olson PN, Maki DD, Griffiths HJ (1997) The prevalence of diffuse idiopathic skeletal hyperostosis (DISH) in two large American Midwest metropolitan hospital populations. Skeletal Radiol 26:222-225

24. Kim SK, Choi BR, Kim CG, Chung SH, Choe JY, Joo KB, Bae SC, Yoo DH, Jun JB (2004) The prevalence of diffuse idiopathic skeletal hyperostosis in Korea. J Rheumatol 31:2032-2035

25. Westerveld LA, van Ufford HM, Verlaan JJ, Oner FC (2008) The prevalence of diffuse idiopathic skeletal hyperostosis in an outpatient population in The Netherlands. J Rheumatol 35:1635-1638

26. Kagotani R, Yoshida M, Muraki S, Oka H, Hashizume H, Yamada H, Enyo Y, Nagata K, Ishimoto Y, Teraguchi M, Tanaka S, Nakamura K, Kawaguchi H, 
Akune T, Yoshimura N (2015) Prevalence of diffuse idiopathic skeletal hyperostosis (DISH) of the whole spine and its association with lumbar spondylosis and knee osteoarthritis: the ROAD study. J Bone Miner Metab $33: 221-229$

27. Julkunen H, Heinonen OP, Pyorala K (1971) Hyperostosis of the spine in an adult population. Its relation to hyperglycaemia and obesity. Ann Rheum Dis $30: 605-612$

28. Mata S, Hill RO, Joseph L, Kaplan P, Dussault R, Watts CS, Fitzcharles MA, Shiroky JB, Fortin PR, Esdaile JM (1993) Chest radiographs as a screening test for diffuse idiopathic skeletal hyperostosis. J Rheumatol 20:1905-1910

29. Ishimoto Y, Yoshimura N, Muraki S, Yamada H, Nagata K, Hashizume H, Takiguchi N, Minamide A, Oka H, Tanaka S, Kawaguchi H, Nakamura K, Akune T, Yoshida M (2017). Association of Lumbar Spondylolisthesis with Low Back Pain and Symptomatic Lumbar Spinal Stenosis in a Population-Based Cohort: The Wakayama Spine Study. Spine 42: E666-E671. 


\section{Table 1}

Characteristics of sample groups at each institution.

\begin{tabular}{lccc}
\hline Variable & Symptomatic LSS patients & $\begin{array}{c}\text { General inhabitants without } \\
\text { LSS }\end{array}$ & p value \\
\hline $\begin{array}{l}\text { Location of } \\
\text { Institution }\end{array}$ & Eniwa, Hokkaido & Wakayama, Kinki & \\
$\begin{array}{l}\text { Investigation } \\
\text { period }\end{array}$ & $2002-2010$ & $2008-2010$ & \\
Total number & 2,363 & 787 & \\
Population & Preoperative LSS Patients & General Population & \\
\hline Male sex, n (\%) & $1,177(49.8)$ & $263(33.4)$ & $<0.001^{*}$ \\
Age, mean (SD) & $68.0(8.4)$ & $67.2(12.4)$ & $0.942^{* *}$ \\
BMI, mean (SD) & $25.0(3.5)$ & $23.2(3.5)$ & $<0.001^{* *}$ \\
DM, n (\%) & $395(16.7)$ & $39(5.0) \dagger$ & $<0.001^{*}$ \\
\hline
\end{tabular}

*Chi square test; **Mann-Whitney U test.

† Two patients were missing data on DM.

LSS: lumbar spinal stenosis; SD: standard deviation; BMI: body mass index; DM: diabetes mellitus 
Table 2

Multivariate analysis of associated factors for symptomatic LSS

\begin{tabular}{lccc}
\hline Variable & $\mathrm{aOR}$ & $\mathrm{p}$ & $95 \% \mathrm{CI}$ \\
\hline Male sex & 1.656 & $<0.001$ & $1.385-1.980$ \\
Age, 1-yr increase & 1.003 & 0.442 & $0.995-1.012$ \\
BMI, 1-unit increase & 1.149 & $<0.001$ & $1.119-1.180$ \\
DM positive & 3.088 & $<0.001$ & $2.183-4.367$ \\
DISH positive & 1.649 & $<0.001$ & $1.317-2.065$ \\
\hline
\end{tabular}

Multiple logistic regression analysis was performed with adjustments for sex, age, BMI, DM, and DISH.

aOR: adjusted odds ratio; CI: confidence interval; BMI: body mass index; DM: diabetes mellitus; DISH: diffuse idiopathic skeletal hyperostosis 


\section{Figure Legend}

2 Fig 1. Prevalence of diffuse idiopathic skeletal hyperostosis (DISH). The prevalence of

3 DISH differed significantly among asymptomatic inhabitant without radiographic

$4 \quad$ lumbar spinal stenosis (LSS) (14.4\%), asymptomatic inhabitant with radiographic

$5 \quad$ severe LSS (21.1\%), and patients with LSS required surgery $(31.7 \%),(\mathrm{p}<0.0001)$.

Fig 1.

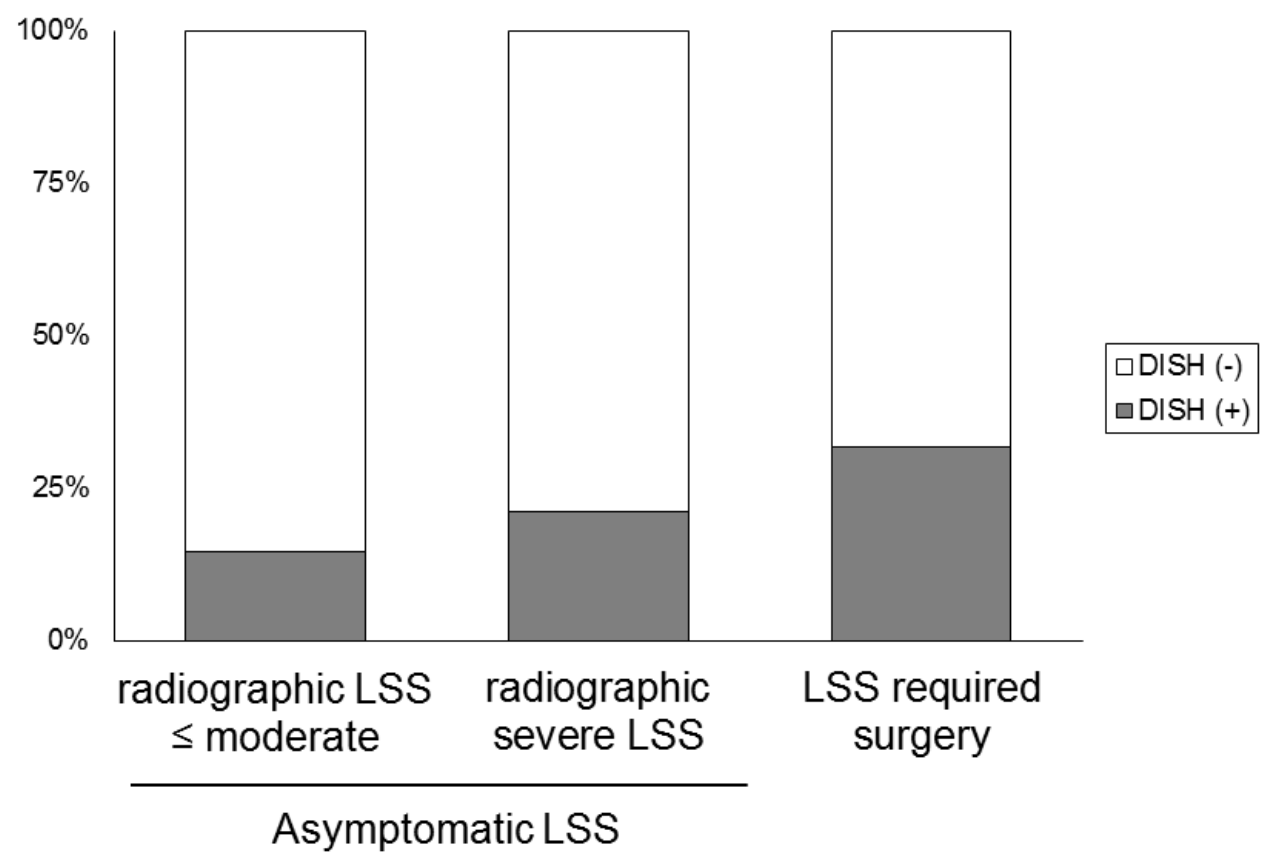

6

7 
1 Fig 2. Distribution of fused vertebrae resulting from diffuse idiopathic skeletal

2 hyperostosis (DISH) at each institution. The box plot represents the sample minimum,

3 the lower quartile or first quartile, the median, the upper quartile or third quartile and

4 the sample maximum for each group. The distribution of DISH was similar, but the

$5 \quad$ median and quartile was more distal in patients with lumbar spinal stenosis (LSS)

6 required surgery than in inhabitants without LSS symptom (T9 vs. T8, respectively, $\mathrm{p}$

$7 \quad \leq 0.001)$. The number of segments fused by DISH was not different between the two

8 groups.

Fig 2.

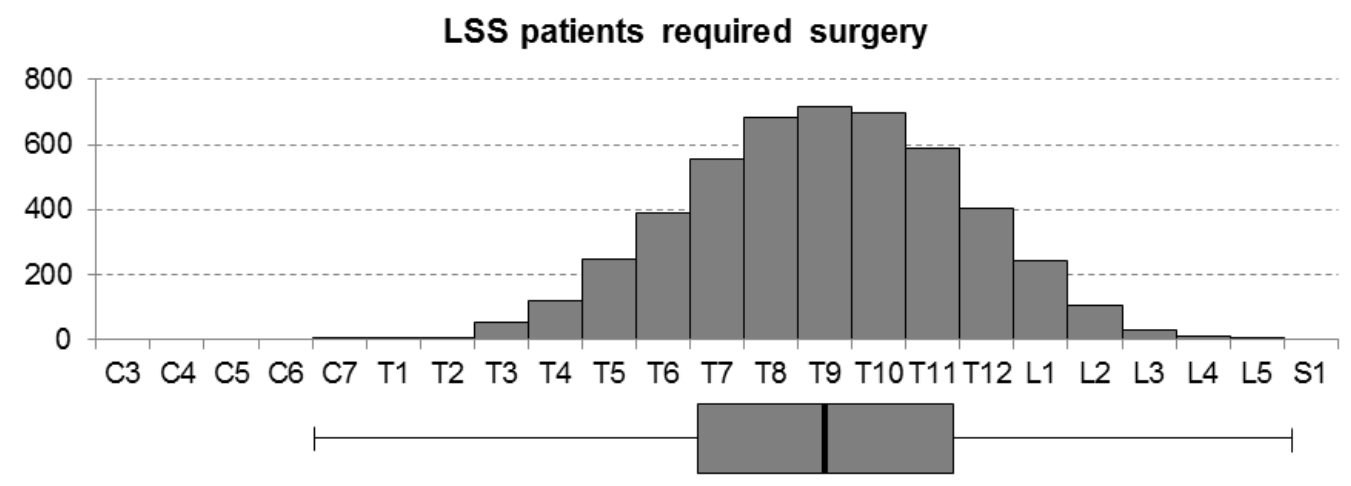

General inhabitants without LSS symptom

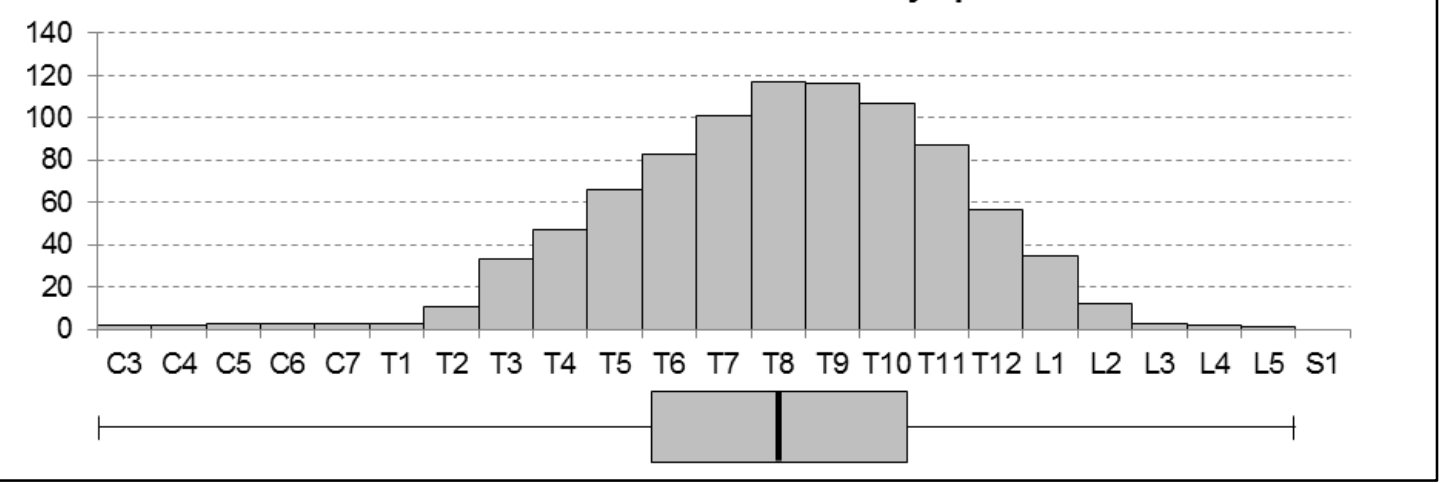


1 Fig 3. (A) Ratio of each lumbar level required decompression between patients with and

2 without DISH. Decompression surgery at L1-2 and L2-3 were more performed in

3 patients with DISH than those without DISH ( $\mathrm{p}<0.001$ ). (B) Distribution of distance of

4 vertebra between lower end of DISH and upper end of decompression level. The just

5 distal segment of DISH (distance $=0$ ) were required decompression in 30 out of 750

6 patients (4\%).

Fig 3.

(A)

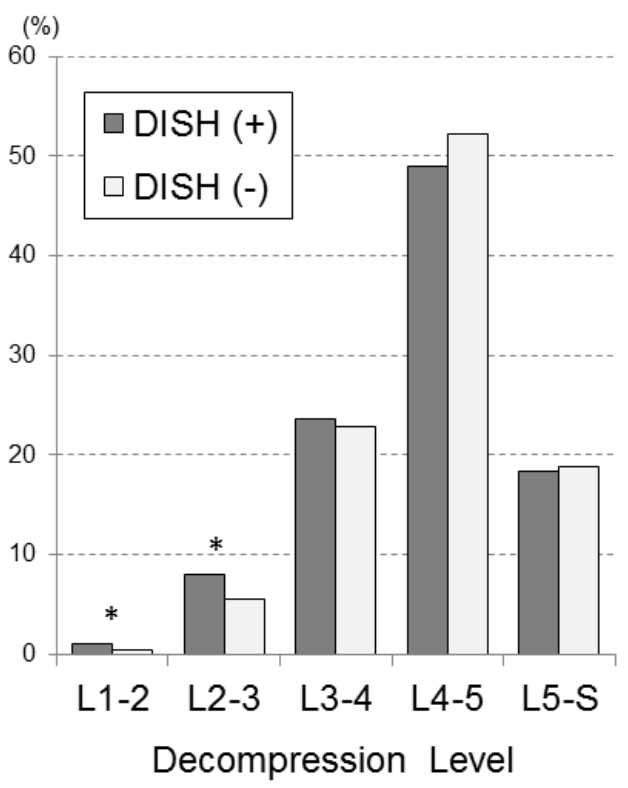

(B)

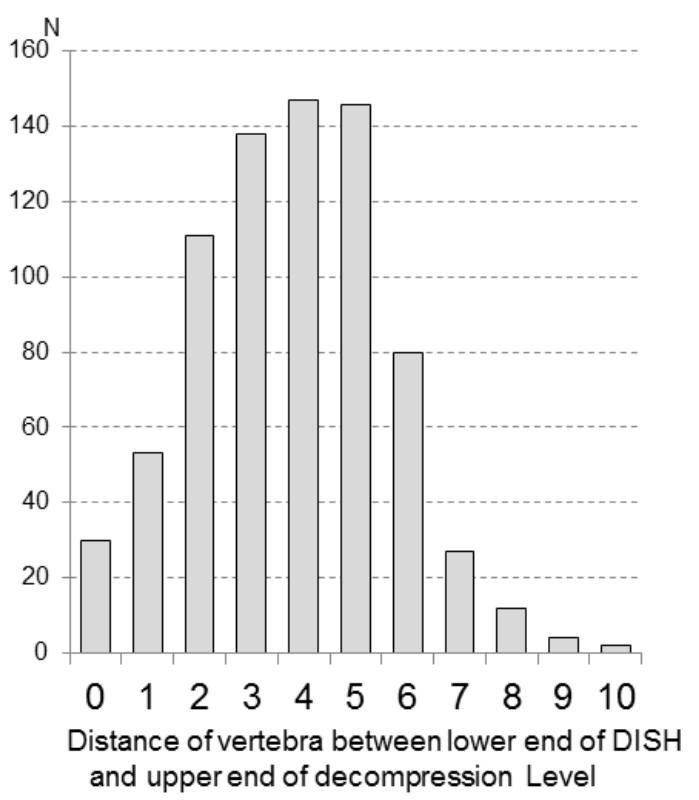

7

8

9

10 Supplemental data

11 Supplemental data 1. (PDF) 


\section{Supplemental Data 1.}

A comparison between lumbar spinal stenosis (LSS) patients requiring surgery in Osaka and general inhabitants without LSS in Wakayama was performed to assess reproducibility in another regional setting. These results were compared with the initial results in the main text. Hokkaido is approximately $1200 \mathrm{~km}$ away from Wakayama; however, Osaka is adjacent to Wakayama (Supplemental Fig 1). The Osaka samples were from our previous report [1]. A total of 132 patients who underwent surgery for symptomatic LSS were included, and the surgical indication was the same as for patients in Hokkaido, in the main text. Also, as in the main text, preoperative whole spine X-rays were used to diagnose diffuse idiopathic skeletal hyperostosis (DISH) by Resnick criteria; height, body weight, body mass index (BMI, $\mathrm{kg} / \mathrm{m}^{2}$ ), and diabetes mellitus (DM) were investigated; and the samples used as general inhabitant without LSS symptom were from the same population.

The prevalence of DISH, sex distribution, and DM were significantly different between the two groups (Supplemental Table 1). Multiple logistic regression analysis of associated factors for LSS requiring surgery was performed with adjustments for sex, age, BMI, DM, and DISH. DISH was an independent associated factor for LSS requiring surgery (adjusted odds ratio, 2.50; 95\% confidence interval, 1.58-3.94) (Supplemental Table 2).

\section{Reference}

1. Yamada K, Toyoda H, Terai H, Takahashi S, Nakamura H (2014) Spinopelvic alignment of diffuse idiopathic skeletal hyperostosis in lumbar spinal stenosis. Eur Spine J 23:13021308 


\section{Supplemental Table 1}

Characteristics of sample groups at each institution.

\begin{tabular}{lccc}
\hline & \multicolumn{2}{c}{ Location of Institution } & p value \\
Variable & Osaka, Kinki & Wakayama, Kinki & \\
\hline Investigation period & $2008-2010$ & $2008-2010$ & \\
Total number & 132 & 787 & \\
Population & Preoperative LSS & General Population & \\
Male sex, n (\%) & Patients & without LSS symptom & \\
Age, mean (SD) & $77(58.3)$ & $263(33.4)$ & $<0.01^{*}$ \\
BMI, mean (SD) & $68.2(10.0)$ & $67.2(12.4)$ & $0.744^{* *}$ \\
DM, n (\%) & $23.8(3.3)$ & $23.2(3.5)$ & $0.056^{* *}$ \\
DISH, n (\%) & $26(19.7)$ & $39(5.0) \dagger$ & $<0.001^{*}$ \\
\hline
\end{tabular}

*Chi square test; **Mann-Whitney U test.

${ }^{\dagger}$ Two patients were missing data on DM.

LSS: lumbar spinal stenosis; SD: standard deviation; BMI: body mass index; DM: diabetes mellitus; DISH: diffuse idiopathic skeletal hyperostosis.

\section{Supplemental Table 2}

Multivariate analysis of associated factors for LSS requiring surgery

\begin{tabular}{lccc}
\hline Variable & aOR & $\mathrm{p}$ & $95 \% \mathrm{CI}$ \\
\hline Male sex & 2.019 & $<0.001$ & $1.344-3.031$ \\
Age, 1-yr increase & 0.992 & 0.398 & $0.975-1.010$ \\
BMI, 1-unit increase & 1.02 & $<0.001$ & $0.965-1.079$ \\
DM positive & 3.857 & $<0.001$ & $2.195-6.776$ \\
DISH positive & 2.497 & $<0.001$ & $1.582-3.939$ \\
\hline
\end{tabular}

Multiple logistic regression analysis was performed with adjustments for sex, age, BMI, DM, and DISH.

aOR: adjusted odds ratio; $\mathrm{CI}$ : confidence interval; BMI: body mass index; DM: diabetes mellitus; DISH: diffuse idiopathic skeletal hyperostosis 
Supplemental Fig. 1. Locations and regions of institutions included in the additional analysis.

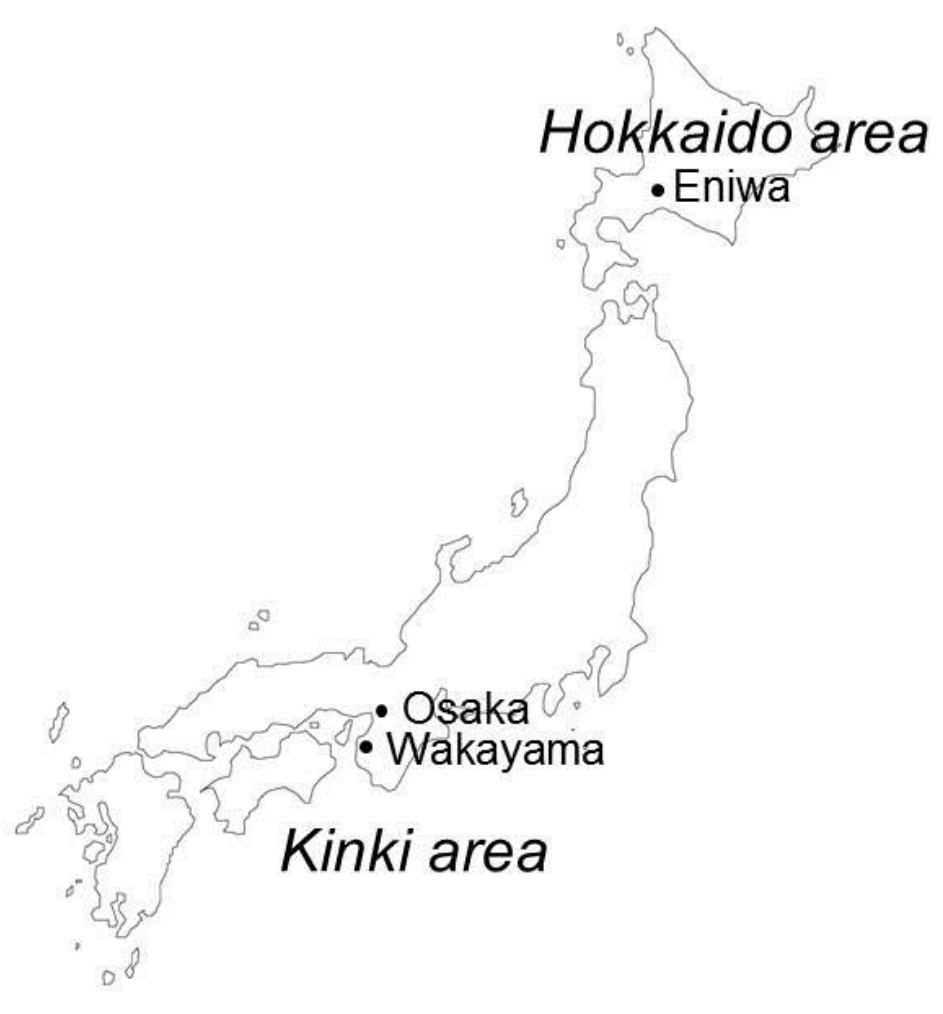

*This supplemental data belongs to Journal of Bone and Mineral Metabolism.

Original article:

Title: Diffuse Idiopathic Skeletal Hyperostosis is Associated With Lumbar Spinal Stenosis requiring surgery

Authors: Kentaro Yamada ${ }^{1,2 *}$, Shigenobu Satoh ${ }^{1}$, Hiroshi Hashizume ${ }^{3}$, Noriko Yoshimura ${ }^{4}$, Ryohei Kagotani $^{3}$, Yuyu Ishimoto ${ }^{3}$, Yuichiro Abe ${ }^{1}$, Shigeyuki Muraki ${ }^{4}$, Hiroaki Nakamura $^{2}$, Munehito Yoshida ${ }^{3}$

1 Department of Orthopaedic Surgery, Wajokai Eniwa Hospital, Hokkaido, Japan

2 Department of Orthopaedic Surgery, Osaka City University, Osaka, Osaka, Japan

3 Department of Orthopaedic Surgery, Wakayama Medical University, Wakayama, Japan

4 Department of Joint Disease Research, 22nd Century Medical and Research Center, Faculty of Medicine, The University of Tokyo, Tokyo, Japan

Corresponding author: Yamada $\mathrm{K}, \mathrm{MD}, \mathrm{PhD}$

E-mail address: yamachen@msic.med.osaka-cu.ac.jp 